\title{
MicroHikari3D: Transformando una impresora 3D en un microscopio robotizado moderno
}

\author{
Pablo T. Toledano, Gloria Bueno, Noelia Vállez, Óscar Déniz, Jesús Salido \\ VISILAB - E.T.S.I. Industriales, Universidad de Castilla-La Mancha, gloria.bueno@uclm.es
}

\begin{abstract}
Resumen
Los microscopios son una herramienta imprescindible para diferentes aplicaciones en biología y medicina, entre otros. La automatización de estos microscopios y la integración de herramientas de análisis automático de imagen es un gran avance en microscopía, pero la mayoría de los sistemas disponibles en el mercado con estas prestaciones tienen un precio elevado. Por otro lado, en los últimos años ha habido un auge importante del desarrollo y venta de impresoras $3 D$ a un precio muy asequible. Por ello, en este trabajo se busca ofrecer un kit modular que permita convertir una impresora $3 D$ en un microscopio motorizado completamente funcional y automatizado. Para conseguir este objetivo, se ha seleccionado una impresora $3 D$, de la que se ha reaprovechado el eje de movimiento cartesiano, y se le ha acoplado un sistema óptico basado en Raspberry Pi HQ Camera, una iluminación superior e inferior y además de una base para portaobjetos nueva. Así como herramientas de procesado y métodos de clasificación automática de la imagen. Con esto se conseguirá un sistema de microscopía modular y de bajo coste, capaz de capturar muestras microscópicas de organismos y tejidos en portas $2 D$ y en placas Petri $3 D$.
\end{abstract}

Palabras clave: microscopio robotizado, procesamiento automático, visión por computador

\section{INTRODUCCIÓN}

La microscopía ha evolucionado desde la creación de los primeros microscopios simples compuestos por un par de lentes y un tubo opaco de H. Janssen y su hijo en 1590, o el fabricado por Galileo Galilei en 1609, hasta complejas máquinas formadas por varias componentes, ópticos y no ópticos [1].

Los microscopios tienen una especial importancia en el avance de la biomedicina, ya que permiten la observación de organismos y elementos de tamaño reducido, imposibles de apreciarse por el ojo humano. Estos elementos van desde $100 \mu \mathrm{m}$ de media, que tienen las células de un tejido humano al rango de $20 \mathrm{~nm}$ - $450 \mathrm{~nm}$ que tienen la mayoría de virus o incluso de $2 \mu \mathrm{m}$ a $200 \mu \mathrm{m}$ que llegan a alcanzar las microalgas. Otros elementos observables por el ojo humano también pueden beneficiarse del uso de un microscopio ya que permite apreciar partes de ellos a mayor aumento y por tanto percibir detalles que se escaparían de otra forma. Por ejemplo, un pelo humano tiene un diámetro medio de $75 \mu \mathrm{m}$; el uso de un microscopio permite analizar la calidad de la fibra capilar pudiendo llegar a observar elementos como la raíz del pelo. Otra utilidad importante es en la industria, por ejemplo análisis de soldadura en circuitos integrados, permitiendo detectar una soldadura rota o dry solder no perceptible a simple vista.

La importancia de automatizar o robotizar estos sistemas y dotarles de métodos de procesado de imagen hace que en los últimos años haya surgido una corriente de implementación de microscopios de bajo coste y con la filosofía de código abierto con el principal objetivo de permitir la democratización de dichos sistemas a precio reducido. Proyectos como Incubot3D [3] utilizaron una impresora 3D para transformarla en un microscopio invertido o OpenFlexure [2] son ejemplos de otros proyectos de microscopía de bajo coste.

Este trabajo describe la adaptación de una impresora 3D para convertirla en un microscopio robotizado totalmente funcional. Los microscopios robotizados incorporan elementos de control como motores paso a paso y computadores, que permiten la digitalización automática de muestras, el autoenfoque o incluso la clasificación por clases de la imagen. Si bien este tipo de microscopios aportan las ventajas anteriores, tienen un inconveniente y no es otro que su elevado precio en comparación con lo que podríamos llamar sistema de microscopía clásicos.

MicroHikari3D nace así como alternativa con el fin de crear un prototipo de coste reducido, modular y con el mayor número de funcionalidades posible. El sistema usa como base la estructura y electrónica de control de una impresora 3D comercial, capaz de adquirir imágenes microscópicas de diferentes tipos de muestras. El microscopio es controlado con una aplicación de móvil. Los obje- 
tivos específicos son:

- Desarrollar un método automático de captura de muestras microscópicas de gran tamaño: el campo de visión (FoV) obtenido con el sistema óptico puede no cubrir toda la muestra que queramos capturar, por lo que se implementará un método de stitching que a partir de varios FoV los ensamble automáticamente.

- Incorporar los elementos necesarios para la toma de imágenes en campo claro y con diferentes longitudes de onda: esto permite observar muestras que requieren del uso de luces con longitudes de onda específicas para poder observarlas.

- Desarrollar un sistema de control basado en API REST: permitir controlar los ajustes de la cámara como el ISO o la resolución, la posición de los motores paso por paso y la iluminación mediante peticiones REST.

- Estudiar el uso de distintos tipos de placas controladoras: ofreciendo un sistema de cómputo que permita alcanzar un equilibro entre velocidad y precio.

\section{HARDWARE}

Los principales componentes hardware del microscopio son: impresora 3D, Raspberry Pi 4, cámara, Arduino UNO, subsistema óptico, iluminación y base para portaobjetos.

La elección de una impresora 3D es fundamental, ya que es la estructura sobre la que se montan el resto de componentes que forman nuestro microscopio (sistema óptico, fuentes de iluminación, cámara ,etc).

\subsection{Impresora 3D}

Hay diversos modelos de impresoras 3D en el mercado. Proyectos como RepRap [4] han permitido que multitud de empresas puedan lanzar su versión de impresora 3D derivada de sus diseños base. En este trabajo se consideraron dos modelos de impresoras: BQ Prusa I3 Hephestos y Tronxy $\mathrm{X} 1$ 3D. Finalmente se seleccionó este último modelo, Tronxy X1 3D (ver Figura 1), sobre la Prusa i3 Hephestos, debido a las siguientes ventajas: tamaño reducido, disponibilidad en el mercado, final de carrera del eje $\mathrm{Z}$ regulable, asa de transporte, construcción más robusta, precio inferior y sobre todo mejor precisión de movimiento. Las precisiones de movimiento de los ejes $\mathrm{X}, \mathrm{Y}$ y Z son por ese orden: $0,012 \mathrm{~mm}, 0,012 \mathrm{~mm}$ y $0,004 \mathrm{~mm}$.

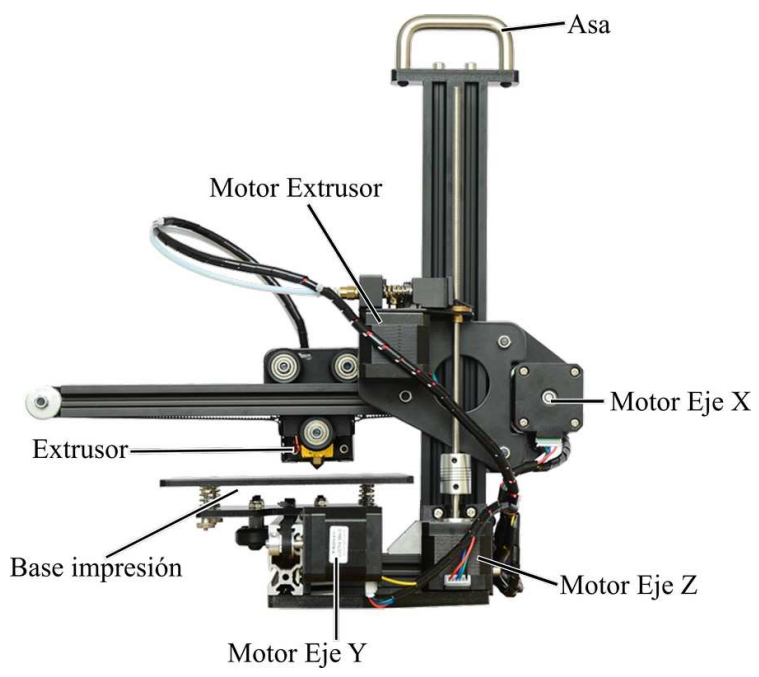

Figura 1: Tronxy X1 3D (Adaptada de Tronxy)

\subsection{Placa de cómputo}

Por otro lado, la plataforma de cómputo elegida para nuestro microscopio fue el ordenador monoplaca Raspberry Pi4. En este trabajo se utilizó la versión 4 del dispositivo. El procesador de esta versión cuarta ha sido actualizado al modelo BCM2711 de la empresa Broadcom, que incorpora cuatro núcleos de arquitectura ARM A72 y una GPU VideoCore VI funcionando a una frecuencia de $1.5 \mathrm{Ghz}$ y $500 \mathrm{Mhz}$ respectivamente. Otro aspecto que ha cambiado en el modelo 4 han sido las configuraciones disponibles de memoria RAM. Mientras que modelos anteriores estaban limitados a un máximo de $1 \mathrm{~Gb}$, la Raspberry Pi 4 puede ser configurada con $1 \mathrm{~Gb}, 2 \mathrm{~Gb}, 4 \mathrm{~Gb}$ o 8 Gb. Aquí hemos usado la configuración con 4 Gb de RAM. Se ha estimado que las únicas operaciones que requieren de grandes cantidades de memoria son las encargadas de hacer el stitching de varios FoV. Esto es así porque los distintos FoV deberán ser almacenados en RAM para su posterior procesado y unión.

En términos de conectividad se usará el puerto Ethernet Gigabit y dos de los puertos USB tenemos a nuestra disposición. Estos puertos serán usados para conectar los dos microcontroladores, el Arduino UNO y el de la impresora 3D elegida. Los anchos de banda proporcionados por estas interfaces satisfacen las necesidades de nuestro microscopio. Por último, la conexión para la cámara puede ser el puerto MIPI CSI de dos vías que incluye.

El sistema operativo elegido será Raspberry Pi OS (anteriormente conocido como Raspbian), una distribución de linux basada en Debian diseñado específicamente para funcionar en SBC Raspberry. 


\subsection{Sistema óptico}

La cámara es un elemento central del microscopio, por lo que su elección es también de gran importancia. Se debe encontrar un equilibrio entre prestaciones, calidad y precio. Como la parte de cómputo de nuestro microscopio estará basado en alguna solución de Single Board Computer, el modelo de cámara elegido deberá utilizar alguno de los protocolos compatibles con la mayoría de ellos. Por ello se ha empleado la Raspberry Pi High Quality Camera, el tercer modelo de cámara para la familia de ordenadores en placa Raspberry Pi. El sensor de esta cámara tienen 12.3 megapíxels. Otros aspectos importantes de este modelo son la incorporación de un sistema de montura con rosca C o CS, que permiten que la cámara se atornille al sistema óptico de nuestro microscopio y un filtro de luz infrarroja. Este filtro puede ser retirado en la situación de que queramos realizar imágenes con fuentes de luz infrarroja, es decir, hacer las funciones de microscopio infrarrojo. Este cambio tendrá que ser realizado teniendo en cuenta que es irreversible.

El Arduino UNO es utilizado en el MicroHikari3D como controlador de los sistemas de iluminación. Esto es gracias a la multitud de pines de entrada y salida, tanto digitales como analógicos disponibles. Para poder establecer la comunicación entre la plataforma de cómputo, la Raspberry Pi 4B, y el microcontrolador ha hecho uso de una conexión serial mediante el puerto USB de ambos. Dentro de este canal se utiliza un lenguaje muy similar al usado por las impresoras 3D, el G-code. Estos comandos accionan las rutinas necesarias para la creación de las señales necesarias para controlar los circuitos de iluminación del microscopio.

El sistema óptico en el MicroHikari3D consiste en todo el conjunto de piezas que permiten a la cámara elegida formar la imagen de la muestra que queramos analizar. Las piezas que lo componen son:

- Lente tubular: lente que permite a los objetivo de aumento enfocar y formar la imagen en el sensor de la cámara. Son especialmente necesarias si se utilizan objetivos plan corregidos al infinito, aunque en los objetivos enfocados a $160 \mathrm{~mm}$ permite acortar la distancia física entre el objetivo y el sensor.

- Objetivo de aumento: componente formado por una o varias lentes que permiten aumentar la imagen.

- Soporte: conjunto de piezas de uno o varios materiales que permiten la unión del objetivo, lente tubular y cámara de tal manera que sea posible que se capture la imagen.
Ha sido necesario diseñar y fabricar piezas 3D en plástico para el agarre de los componentes. El resultado de acoplar todas las piezas del listado anterior se puede observar en la Figura 2.

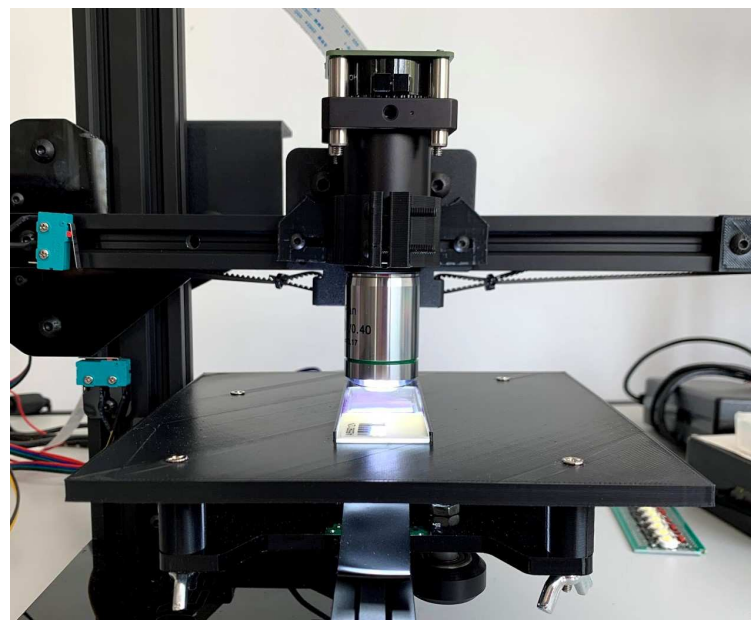

Figura 2: Montaje óptico metálico acoplado al MicroHikari3D

\subsection{Sistemas de iluminación}

La iluminación es una de las partes más importantes de un microscopio. Los modos de iluminación qué tenga disponible definirán con qué tipo de microscopio estamos trabajando. El MicroHikari3D pertenece a la categoría de microscopios de campo claro. En ellos, los rayos de luz originados por el sistema de iluminación atraviesan o son reflejados por el espécimen, sin ser estos alterados más allá de los cambios que pueda hacer la propia muestra.

Se han diseñado dos sistemas de iluminación distintos, uno inferior encargado de generar luz que atraviese la muestra y otro superior encargado de generar luz que sea reflejada por la muestra. El uso de un sistema u otro dependerá de la naturaleza de la muestra observada. Una muestra fina y translúcida es perfecta para sistema de iluminación inferior, mientras que para muestras más gruesas u opacas el sistema de iluminación superior es el idóneo.

El control de la iluminación se realiza con la placa ya mencionada, el Arduino UNO, a la que se le ha acoplado un shield o escudo, son unas placas con electrónica que se conectan por la parte superior del Arduino y hacen las funciones de placa de expansión. Este shield se ha construido con una placa $\mathrm{PCB}$ de prototipado de $6 \mathrm{~cm} \mathrm{x} 4 \mathrm{~cm}$ a la que se le ha soldado unos pines de tal manera que pueda ser insertada en los conectores macho del Arduino. Unos conectores con tornillos permiten la rápida conexión con las fuentes de alimentación conmutadas CC a CC y con los sistemas de ilumi- 
nación. El circuito compuesto por relé, transistor y resistencia permite controlar la alimentación de la iluminación inferior. La Figura 3 muestra como quedan los componentes electrónicos ya montados en el shield y este acoplado junto al Arduino UNO en el MicroHikari3D.

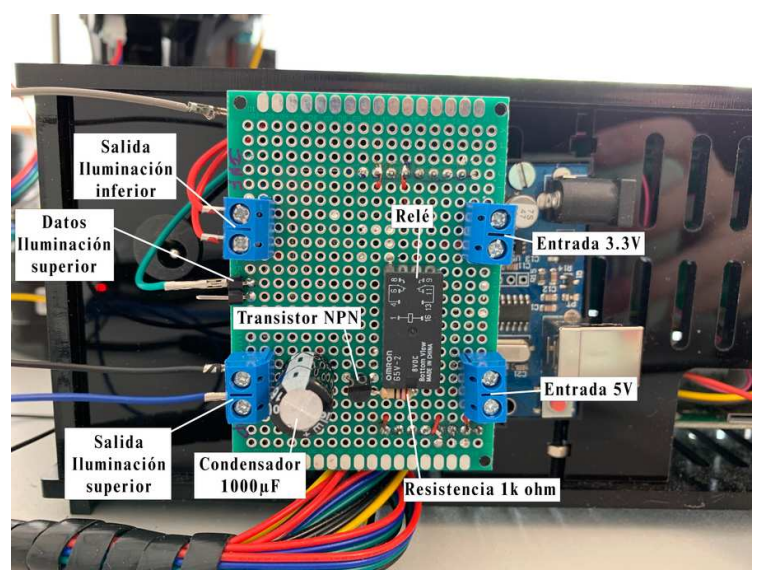

Figura 3: Shield de control de la iluminación

Otro aspecto importante es la alimentación de ambos sistemas de iluminación. Para este fin se ha optado por utilizar la fuente de alimentación de la impresora 3D y aprovechando que ya no existe el consumo que tendría el extrusor se puede derivar esa corriente a otros usos como la iluminación.

El sistema de iluminación inferior es el encargado de proyectar rayos de luz que atravesarán la muestra, idóneo para las muestras especialmente finas. Se ha optado por no poner un sistema fijo, ya que puede surgir que en un caso de uso se necesite un tipo especifico de iluminación por ejemplo, luz ultravioleta o una disposición de LED determinado. Es por esto que se ha optado por un diseño modular que permite deslizar unas placas con la configuración de LED y longitud de onda que se vea necesario. Estas placas son unas PCB de prototipado, parecidas a la usada para el shield de control, pero de tamaño $7 \mathrm{~cm} \mathrm{x} 3 \mathrm{~cm}$. En las placas se pueden soldar los LED que se quieran en la disposición que se estime. La Figura 4 enseña varias de estas placas con configuraciones diferentes.

La sujeción de estas placas la realiza una pieza de plástico (Figura 5) que cuenta con una hendidura donde se deslizarán. Al final de esta pieza se encuentra un conector hembra que hace contacto con su equivalente macho soldado en la placa PCB. La parte superior de la pieza permite el deslizamiento de un difusor de luz o de un filtro. El shield de control permite que las luces de este sistema se puedan encender y apagar de forma remota.

El sistema de iluminación superior es el encargado de proyectar rayos de luz que serán reflejados por la muestra. Es utilizado en muestras que tienen un

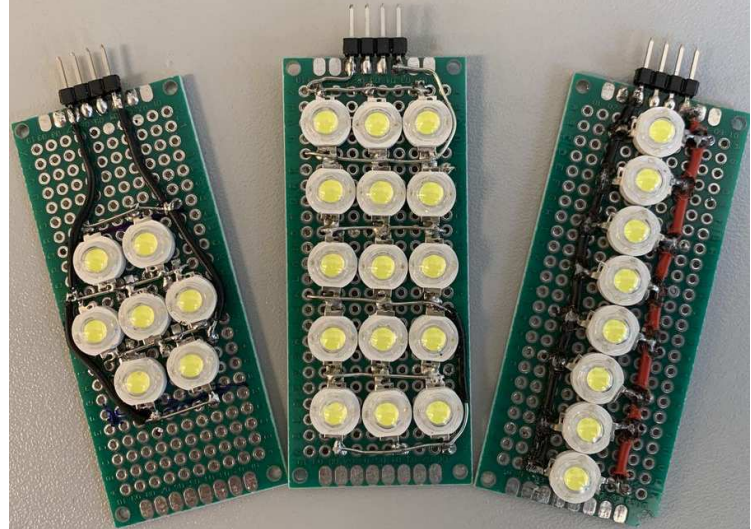

Figura 4: Placas modulares del sistema de iluminación inferior

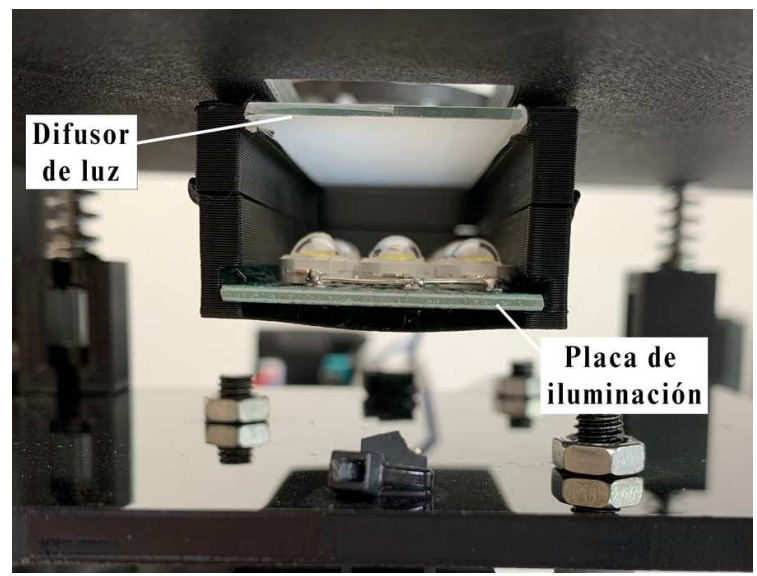

Figura 5: Sistema de iluminación inferior

grosor elevado o que son opacas.

Se compone de dos partes: Aro de luz NeoPixel de la compañía Adafruit y Pieza de plástico que lo mantiene unido al sistema óptico. El aro de luz NeoPixel está formado por 12 LED RGBW de tipo $5050 \mathrm{SMD}$ con brillo elevado ordenados en un círculo con $37 \mathrm{~mm}$ de diámetro. La luz RGB permite elegir el color específico o lo que es lo mismo, elegir que longitud de onda queremos emitir sobre la muestra. La parte blanca emite un luz con temperatura de $4500 \mathrm{~K}$ considerada luz blanca natural.

El control se realiza con señales de tipo modulación por ancho de pulsos (PWM) de 8 bits por canal, como los LED tienen cuatro canales (RGB y W) la suma teórica total equivale a color de 32 bits. Cada LED del NeoPixel esta equipado con un microcontrolador WS2812B, este chip se encarga de regular la intensidad de cada canal de acuerdo a la señal que reciba por el pin de datos. A diferencia de otro tipo de microcontroladores, el control del WS2812B solo requiere de una única señal. Esto es así gracias al uso de registros de 
desplazamiento, ahorrándonos el uso de más cables de los necesarios. Las señales de control son producidas por el microcontrolador Arduino UNO mediante el uso de la biblioteca NeoPixels publicada por el fabricante.

\subsection{Placa para portaobjetos}

Finalmente, para la platina, base donde se colocan las muestras a observar y digitalizar, hemos sustituido la base donde originalmente se extruía el plástico en la impresora Tronxy 3D por una impresa en PLA adaptada a nuestro caso de uso. Los portaobjetos siguen el estándar DIN ISO 8037-1 con medida estándar de $76 \mathrm{~mm}$ x $26 \mathrm{~mm}$ x 1,5 $\mathrm{mm}$. Para montar esta base se han incorporado una serie de elevadores con la finalidad de aumentar el espacio disponible entre la pieza conectada a la correa del eje y la base portaobjetos. Ese espacio ganado permite la incorporación del sistema de iluminación inferior sin eliminar la posibilidad de ajustar la nivelación de ésta. Tener una base lo más nivelada posible es importante especialmente para aumentos altos (superiores a 40x) con el fin de reducir aberraciones en los bordes de la imagen.

\section{SOFTWARE}

El software del MicroHikari3D está basado en el modelo cliente-servidor de dos capas. La Figura 6 muestra la arquitectura y conexiones del sistema. En esta arquitectura el encargado de implementar las funciones de lógica de presentación, tales como las interfaces de usuario, es el cliente mientras que la parte dedicada a la lógica de negocio y datos se reserva para el servidor. El modelo cliente-servidor se refleja en el software del MicroHikari3D en sus dos principales elementos:

1. Servidor: programa escrito en Python 3 encargado de proporcionar el vídeo en directo de la cámara, la captura de imágenes, el control de los motores paso a paso, el control de los sistema de iluminación, etc.

2. Cliente: aplicación para dispositivos móviles Android desarrollada con Flutter donde se puede visualizar el vídeo en directo enviado por el servidor, crear las peticiones de movimiento de los motores paso a paso, encendido de los sistema de iluminación, etc.

\subsection{Servidor}

El servidor del sistema se ejecuta en la plataforma de cómputo Raspberry Pi 4, y está encargado de proveer los servicios que definen el microscopio. A continuación se describen las bibliotecas habilitadas en el servidor de MicroHikari3D:

- Open $C V$ : conjunto de más de 2500 algoritmos optimizados dedicados a métodos de visión por computador.

- Flask: framework web en Python usado para proporcionar al servidor capacidad HTTP.

- Flask-RESTful: extensión de Flask. Añade soporte para la programación de APIs REST.

- PiCamera: interfaz de Python que permite interactuar con las cámaras creadas por la fundación Raspberry.

- Logging: biblioteca usada para registrar los mensajes de depuración durante el desarrollo.

- Unittest: módulo de desarrollo de pruebas unitarias en Python utilizado para probar la API REST.

- TensorFlow Lite: framework de la familia de TensorFlow más ligero y restringido ideado para realizar inferencia en dispositivos móviles o IoT.

Uno de estos servicios es la emisión de vídeo en vivo. Para ello se ha utilizado la función gen del framework Flask para crear una emisión MJPEG. En la compresión MJPEG cada uno de los fotogramas enviados del vídeo se codifica como una imagen JPEG.

El control de los ajustes de la cámara, posición y movimiento de los motores paso a paso y de los sistemas de iluminación se realiza mediante una API REST. El servidor del MicroHikari3D utiliza archivos JSON para representar los estados de los motores paso a paso, la iluminación y la cámara.

\subsection{Procesamiento de la imagen}

Uno de los principales problemas a la hora de capturar imágenes con un microscopio (de cualquier clase) es que la imagen puede quedar desenfocada por secciones al tener distintos planos de enfoque. Esto se traduce en que distintas partes de la imagen se encontrarán enfocadas a distintos valores del eje Z. Para conseguir una imagen enfocada, se ha incluido en el servidor la opción de realizar un apilamiento de enfoque o focus stacking. El apilamiento de enfoque (ver Figura 7) consiste en fusionar o unir varias fotografías obtenidas a distintos planos focales (valores del eje Z) teniendo como resultado una imagen final en la que los valores de sus píxeles serán los del plano focal donde estén más definidos. En el servidor del MicroHikari3D 


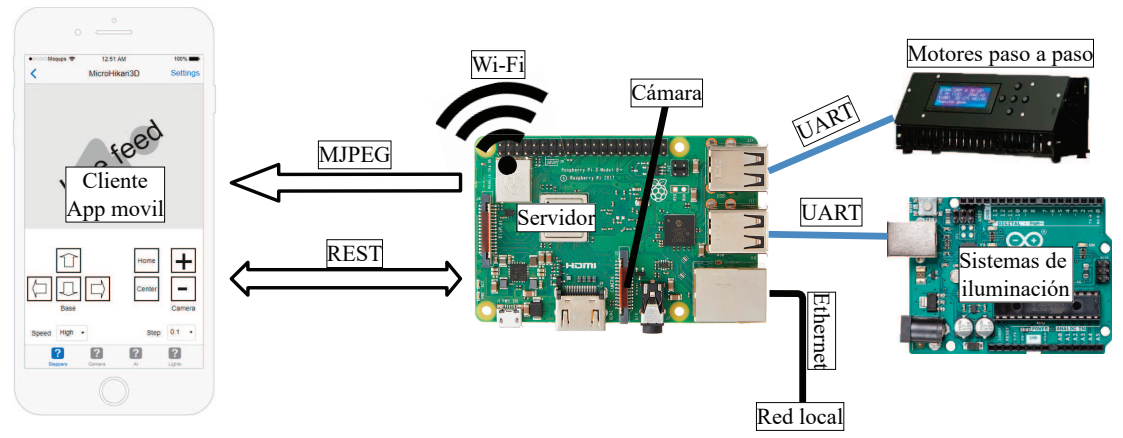

Figura 6: Arquitectura y conexiones del MicroHikari3D



(a) Muestra 1



(c) Muestra 3

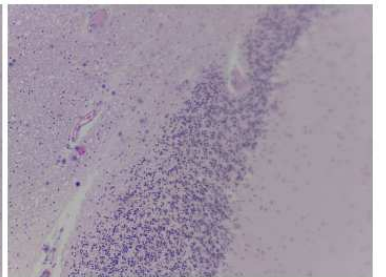

(b) Muestra 2



(d) Resultado
Figura 7: Apilamiento para realizar el enfoque en el MicroHikari3D

se incluye la posibilidad de hacer apilamiento de enfoque de 3,5 o 7 muestras.

La función de escaneo automático permite pedirle al servidor que realice el stitching de varios FoV. Esta herramienta es muy útil para obtener una Whole Slide Image (WSI). El área de cada FoV está definido por el tipo de objetivo de aumento que se utilice. Esto quiere decir que la zona digitalizada con un objetivo de 20 aumentos (20x) no es la misma que con uno de 10 aumentos (10x), aunque la resolución de la imagen sea exactamente la misma. El proceso de escaneo automático del MicroHikari3D es posible gracias a la integración en el servidor de la utilidad de stitching xy-stitch ${ }^{1}$.

El enfoque automático es el algoritmo que se encargará de realizar las funciones que en un microscopio óptico clásico realizarían los diales macrómetro y micrómetro, moviendo el eje $\mathrm{Z}$ hacia arriba o hacia abajo hasta un punto en el que se pueda decir que la imagen formada en el sensor de la cámara se encuentra enfocada. Para medir el nivel de enfoque en una imagen se ha optado por apli-

\footnotetext{
${ }^{1}$ https://github.com/JohnDMcMaster/xystitch
}

car la varianza del Laplaciano como propuso J. L. Pech-Pacheco et al.[5].

Una de las posibilidades que da la integración de un computador directamente en el microscopio es la aplicación de algoritmos de procesado e inteligencia artificial. Así mediante el uso de TensorFlow Lite se han integrado modelos de clasificación de tejidos que permite discernir entre zona maligna o benigna para cada FoV. Para ello se realizan los siguientes pasos:

1. El cliente realiza la petición de realizar inferencia con un modelo determinado.

2. El servidor comprueba que el modelo pedido se encuentra en el JSON de modelos disponibles y carga los datos necesarios: localización, etiquetas de las clases y nombre.

3. El servidor captura una imagen de lo que se está mostrando por la cámara.

4. El servidor carga el modelo de TensorFlow Lite y preprocesa la imagen para adecuarla a la capa de entrada del modelo: redimensionado, cambio de canales, etc.

5. El servidor realiza el proceso de inferencia con la imagen obtenida en el paso anterior, etiqueta los resultados obtenidos de la inferencia y envía la respuesta al cliente.

6. El cliente recibe los resultados y los muestra como vea conveniente.

Para la carga de modelos se ha creado un JSON donde se pueden añadir los modelos que se quieran usar en el servidor. Además, una petición HTTP de tipo GET a /inference sin el apartado de consulta o query permite conocer los modelos que se encuentran en dicho JSON. Por el contrario, si la petición se realiza con uno de esos modelos en el apartado de query, entonces se realiza el método de inferencia sobre ese modelo.

En este trabajo se han entrenado cuatros modelos de clasificación de imágenes para la detección 
de tejidos cancerígenos en biopsias de aguja fina. Los modelos entrenados han sido: MobileNetV2, EfficientNet0 Lite, ResNet50 e InceptionV3. Estos modelos fueron entrenados en un sistema con mayores capacidades de cómputo y fueron posteriormente incorporados al servidor. Los modelos se pueden usar para mostrar la clase con mayor probabilidad dentro de la aplicación para dispositivos móviles como muestra la Figura 8. Esto es especialmente útil en entornos médicos, aunque también puede extenderse a otros como el estudio de la calidad del agua, donde pueden clasificarse microorganismo como las diatomeas.
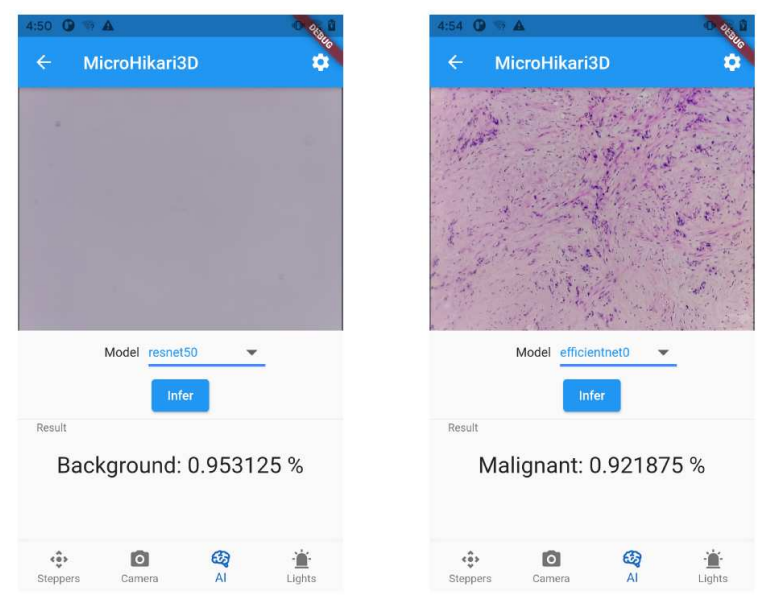

Figura 8: Aplicación para móviles mostrando el resultado de la clasificación

El Arduino UNO es el sistema basado en microcontrolador encargado de la gestión de los sistemas de iluminación, por lo que es necesario especificar algún tipo de protocolo de comunicación entre él y el programa servidor. Esto significa por ejemplo que una petición de encender la iluminación inferior realizada por la aplicación de móvil llegará al servidor en la plataforma de cómputo y éste deberá transmitirla al Arduino para que el relé se accione. Para ello se ha optado por conectar la plataforma de cómputo, Raspberry Pi 4 y el Arduino UNO con un cable USB, aprovechando así el convertidor USB-UART que tiene incorporado.

La aplicación para dispositivos móviles permite de forma ágil el control de las funciones del microscopio. Para el desarrollo de la aplicación, se ha utilizado el conjunto de herramientas de desarrollo Flutter de Google. La aplicación está divida en dos partes: la parte superior, donde se muestra el vídeo en directo de la cámara del microscopio, y la parte inferior, donde aparecen los paneles de cada una de las funciones incorporadas. Los paneles incluidos en la aplicación son: Panel de Steppers (controla los motores paso a paso), Panel Camera (con botones de captura de imágenes normales, con apilamiento de enfoque y panorámicas), Panel
AI (clasificación del FoV mostrado por la cámara del microscopio) y Panel Lights (controles de los sistemas de iluminación).

Además de los paneles mencionados anteriormente, también se dispone de una pantalla de configuración donde poder cambiar ajustes como: resolución de las imágenes, resolución de la emisión en directo, filtro aplicado a las imágenes, ISO, intensidad del filtro de sharpness o nitidez y la saturación de la imagen.

\section{RESULTADOS}

A continuación se muestran imágenes adquiridas con el sistema MicroHikari3D descrito. El tiempo en adquirir imágenes de $1 \times 1$ FoV es de 3 segundos y para $3 \mathrm{x} 3$ FoVs, varía entre 2 y 12 minutos para una resolución de $3886 x 2885$ y $9670 x 7137$ píxeles, respectivamente, incluyendo el apilamiento para hacer enfoque completo de los FoV.

Los modelos de clasificación de imágenes probados obtuvieron la precisión y tiempos totales de inferencia mostrados en la Tabla 1.

Tabla 1: Precisión y tiempo de inferencia de los modelos probados en MicroHikari3D

\begin{tabular}{|l|l|l|}
\hline Modelo & Precisión & Tiempo \\
\hline MobileNetV2 & $0.91915 \%$ & $128 \mathrm{~ms}$ \\
EfficientNet0 Lite & $0.92137 \%$ & $159 \mathrm{~ms}$ \\
ResNet50 & $0.92469 \%$ & $900 \mathrm{~ms}$ \\
InceptionV3 & $0.92248 \%$ & $1090 \mathrm{~ms}$ \\
\hline
\end{tabular}

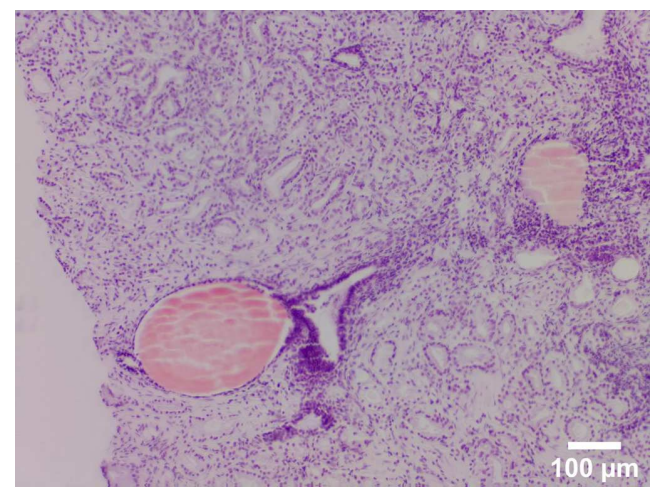

Figura 9: Muestra patológica capturada a 20x

\section{CONCLUSIONES}

Se ha presentado un sistema de microscopía de bajo coste implementado a partir de una impresora 3D. La capacidad del sistema se ha ilustrado con imágenes digitalizadas de muestras biológicas y médicas; y la integración de algoritmos de inteligencia artificial que permiten clasificar las mues- 




Figura 10: Copépodo en medio acuoso a 20x

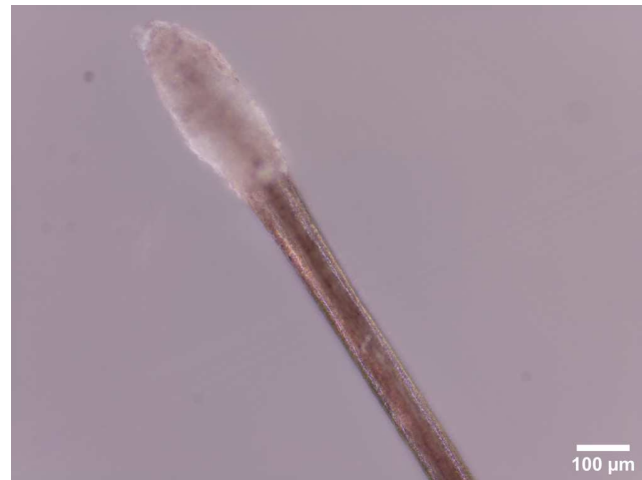

Figura 11: Pelo humano a 20x

tras mediante el uso del popular framework TensorFlow Lite. Como futuro trabajo se evaluará la calidad de las imágenes digitalizadas mediante diferentes métricas y se optimizará el tiempo de adquisición de la WSI.

\section{Agradecimientos}

Los autores agradecen financiación de la Junta de Comunidades de Castilla-La Mancha con el proyecto SBPLY/19/180501/00 0273 y a la Universidad de Castilla-La Mancha por la financiación al grupo de investigación VISILAB.

\section{English summary}

\section{MicroHikari3D: Transforming a 3D printer into a modern robotic micros- cope}

\section{Abstract}

Microscopes are an essential tool for different applications in biology and medicine, among others. The automation of these microscopes and the integration of automatic image analysis tools is a breakthrough in microscopy. However, most of the systems available on the market with these features have a high price, which prevents them from becoming more popular. On the other hand, in recent years there has been a significant boom in the development and sale of $3 D$ printers at a very affordable price. Therefore, this work seeks to offer a modular kit that allows converting a $3 D$ printer into a fully functional and automated microscope. To achieve this goal, all possible parts of a commercially available $3 D$ printer will be used, to which all the necessary optical and lighting elements will be attached. This will result in a modular and cost-effective microscopy system.

Keywords: robotic microscope, automatic processing, machine vision.

\section{Referencias}

[1] R. M. Sánchez Lera y N. R. Oliva García, Historia del microscopio y su repercusión en la Microbiología, Humanidades M, vol. 15, págs. 355-372, ago. de 2015, ISSN: 1727-8120.

[2] J. T. Collins y col., Robotic microscopy for everyone: the OpenFlexure Microscope, bioRxiv, 2019. doi: 10.1101/861856.

[3] G.O.T. Merces y col., The Incubot: A 3D Printer-Based Microscope for Long-Term Live Cell Imaging within a Tissue Culture Incubator, HardwareX, e00189, 2021, ISSN: 24680672. doi:10.1016/j.ohx.2021.e00189.

[4] R. is a community project. RepRap website. (22 de mar. de 2021), [En línea] URL: https: //reprap.org/wiki/RepRap

[5] Pech-Pacheco, J.L. and Cristobal, G. and Chamorro-Martinez, J. and FernandezValdivia, J.Diatom autofocusing in brightfield microscopy: a comparative study, Proceedings 15th International Conference on Pattern Recognition, vol. 3, doi: 10.1109/ICPR.2000.903548.

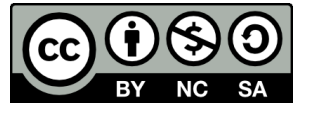

(C) 2021 by the authors. Submitted for possible open access publication under the terms and conditions of the Creative Commons Attribution CC BY-NC-SA 4.0 license (https://creativecommons.org/licenses/by-ncsa/4.0/deed.es). 